\title{
APOPTOTIC EFFECT OF LONG-TERM ADMINISTRATION OF ANTIDEPRESSANT DULOXETINE ON THE TONGUE OF ALBINO RATS: HISTOLOGICAL AND IMMUNO-HISTOCHEMICAL STUDY
}

\author{
Mervat M. Youssef*, Ahmed M. Korraah**and Enas M. Hegazy*
}

\begin{abstract}
Introduction: Depression is one of the most common causes of loss of health and productivity and inability to work. Duloxetine (Cymbalta) is an antidepressant drug that belongs to the class of Serotonin-norepinephrine reuptake inhibitors (SNRIs), which is used for the treatment of major depressive disorder (MDD). In addition, this class is used for the treatment of anxiety disorders. One of the members of the bcl-2 family is the BAX (BCL2 Associated X, Apoptosis Regulator) and it has a key role in the mitochondrial apoptotic process. Under stress conditions, Bax undergoes a conformation change that triggers apoptosis.
\end{abstract}

Objectives: This study aimed to examine the possible impact of long term administration of antidepressant duloxetine on the oral mucosa and salivary glands in tongue.

Material \& methods: Twenty adult male albino rats were divided into two equal groups. Group one served as control group. Group two served as treated group which received duloxetine dissolved in distilled water in a daily oral dose of $10 \mathrm{mg} / \mathrm{kg}$ body weight using a curved metallic oro-pharyngeal tube. At the end of the experiment which lasted for three months, the rats were euthanized by cervical dislocation. Tissue samples were fixed in $10 \%$ formalin, embedded in paraffin, and stained with hematoxylin and eosin to evaluate histopathological finding. Masson' trichrome for collagen detection. Other sections were used to reveal the immuno-histochemical expression of Bax antibodies through computerized image $\mathrm{j}$ analysis software. The expression of the marker was analyzed statistically using Mann-Whitney test.

Results: Histopathological finding of long term administration of duloxetine treated animals showed noticeable atrophic and degenerative changes in the surface epithelium and lamina propria of both the dorsal and ventral surfaces of the tongue as well as the lingual salivary gland. Immunohistochemical finding revealed marked increase in the expression of Bax all over the epithelial cells as well as fibroblast, blood vessels and the lingual salivary glands in the lamina propria. Statistical finding in duloxetine treated animals showed highly significant difference in the expression of Bax.

Conclusion: Although duloxetine being useful drug in treatment depression, anxiety and severe pain, it harmfully affected the histological structure of oral mucosa and salivary glands of the tongue in adult rats. Accordingly using of these drugs should be under supervision and strong caution.

KEY WORDS: Antidepressant, Apoptosis, Bax, Duloxetine, Tongue.

* Assistant Professor of Oral Biology, Faculty of Dentistry, Suez Canal University.

** Lecturer of Oral Pathology, Faculty of Dentistry, Suez Canal University. 


\section{INTRODUCTION}

Depression is one of the most common causes of loss of health and productivity and inability to work. The disease leads to a stark decrease in quality of life for those afflicted and their nearest ones. Mental Disorders' diagnostic and Statistical Manu$\mathrm{al}^{(1)}$ provides a description of depression's emotional symptoms including the lack of interest, sadness, thoughts of suicide, and guiltiness. There is a wide recognition of the physical symptoms as one of the depressive syndrome's primary elements. Such elements entail the following: changes in appetite, changes in psychomotor function, headaches, pain, sleep disorders, the lack of energy, and gastrointestinal disorders.

There are several forms of depression; seasonal affective disorders (SAD) and bipolar depression. From these include major depressive disorders (MDD), dystrophic disorders, psychotic depression, postpartum all MDD and dystrophic disorder are the most common.

There is a hypothesis stating that the biological etiology of depression is the shortage of monoaminergic neurotransmitters, namely the shortages of dopamine and serotonine (5-hidroxitriptamina; $5-\mathrm{HT}$ ) and norepinephrine (NE) ${ }^{(2)}$. Belmaker and Agam $^{(3)}$ showed that the location of the majority of the dopaminergic, noradrenergic and serotonergic (monoaminergic) neurons is in midbrain and brainstem nuclei. In addition, they are projected to huge areas of all over the brain. Such anatomy makes a suggestion that the monoaminergic systems have a major role in regulating a wide range of functions of brain including cognition, appetite, sleep, attention and mood. It has been proven that approximately every compound, which has the ability to inhibits monoamine reuptake resulting in an increase in monoamines' concentration in the synaptic cleft, is considered an antidepressant with high clinical effectiveness. Furthermore, there are antidepressant effects created by the inhibition of the enzyme monoamine oxidase, which causes an increase in monoamines' availability in presynaptic neurons. The theory of depression, which is most pharmacologically relevant, known as the monoamine-deficiency hypothesis, was been developed based on those observations.

There are number of methods to treat depression and anxiety. Medicines are an alternative, psychotherapy such as cognitive behavioral therapy (CBT) is another. In pharmaceutical treatment there are different classes of medicines to choose between. The first discovered class of drugs for treatment of depression is tricyclic antidepressants(TCAs). Its action is mainly by inhibiting uptake of serotinin and norepinephrine and lesser extent to dopamine reuptake. The key short coming related to the TCAs is that there is a huge number of side effects which will lead to discontinuation of treatment.These side effects are due to the non selective binding to various receptors. Also the overdose may lead to severe cardiac conduction abnormalities or arrhythmias. For such reasons, the use of TCAs is restricted ${ }^{(4)}$.

Another class of antidepressant is introduction of selective serotonin reuptake inhibitors (SSRIs) which stand for more than half of the sales of antidepressants. They are described as being selective due to their major effect on serotonin (5-hidroxitriptamina; 5-HT), not on other neurotransmitters. They have the ability to mitigate moderate to severe depression symptoms. Further, they have relative safety and typically result in fewer side effects compared toTCAs. Moreover, through increasing serotonin levels in the brain by inhibiting serotonin reabsorption (reuptake), resulting in more serotonin availability, the SSRIs are able to mitigate depression. However, they can have severe side effects including delayed ejaculation, anorgasmia, and sexual dysfunction and there could be a decrease in libido in about $60 \%$ of patients ${ }^{(5,6)}$,sleep disturbances, anorexia ${ }^{(6)}$, constipation and dry mouth ${ }^{(7)}$. 
A new class of SSRI antidepressants that act by inhibition of the uptake of noradrenaline and serotonin neurotransimitters, allow more effective control of emotional and physical symptoms of depression and are the drug of choice for the treatment of depression because of their higher efficiency, less side effects and good tolerability as compared to the other classes of antidepressants.

The category of antidepressant drugs, which is used for the treatment of major depressive disorder (MDD) and possibly for the treatment of anxiety disorders, is the SNRIs (Serotonin-norepinephrine reuptake inhibitors). According to Cashman and Ghirmai ${ }^{(8)}$, dually inhibiting serotonin and norepinephrine reuptake has several advantages compared to other antidepressant drugs as it treats a broad range of symptoms.

A part of this category is the duloxetine (Cymbalta), which was proven in August 2004(9) to treat depression and neuropathic pain. In addition, it was reported to treat fibromyalgia ${ }^{(10)}$, diabetic neuropathy, major depressive disorder (MDD), and generalized anxiety disorder (GAD) ${ }^{(11,12)}$. An enormous success in treating the chronic musculoskeletal pain, which includes chronic low back pain and chronic osteoarthritis pain, has been recently shown by duloxetine $^{(13)}$. Furthermore, there is a putative relationship between its analgesic efficacy in the center of the brain and its effect on descending inhibitory pain pathways.

In case of severe dental and paradental pain, there is a recommendation to use duloxetine (Cymbaltal) in dentistry. Using duloxetine as the first-option medication to treat burning mouth syndrome (BMS) was indicated by some reports ${ }^{(14,15)}$.

A confirmation is provided by Nagashima et $\mathrm{al}^{(16)}$ that the duloxetine can significantly mitigate chronic nonorganic oro-facial pain. After two weeks of treatment, its effect of reducing the pain started to appear. Moreover, whether the baseline depressive symptoms are present or absent, duloxetine effects of mitigating the pain have appeared.
There is a relationship between most prescribed antidepressant medications and the number of significant oral reactions, which include sialoadenitis, dysgeusia, xerostomia, tongue oedema with discoloration, glossitis, and stomatitis. As a result of salivary gland dysfunction caused by the medication, these oral reactions appear. The decrease in the production of saliva can be a cause of taste alteration and burning sensation. ${ }^{(17)}$

The key characteristics of Apoptosis or programmed cell death are the distinct morphological features and the mechanisms which are biochemically dependent on energy. Normally, the apoptosis occurs during the period of growth and aging. It occurs as a homeostatic mechanism for maintaining the populations of cells in tissues ${ }^{(18)}$ and as a defensive mechanism as in the case of immune reactions or in case of cell damage due to medical, environmental or disease toxicities ${ }^{(19)}$.

One of the members of the bcl-2 family is BAX (BCL2 Associated X, Apoptosis Regulator) which has an important role in the mitochondrial apoptotic process. Under usual circumstances, it mostly becomes cytosolic through persistent retrotranslocation to the cytosol from mitochondria where the BCL2L1/Bcl-xL, which is characterized by the avoidance of toxic BAX level accumulation at the mitochondrial outer membrane ${ }^{(20)}$. Bax forms heterodimers with bcl-2, in which its distribution is inversely related to that of bcl- $2^{(21)}$. Thus, the bcl-2/ bax ratio controls the relative susceptibility of cells to stimuli, which induce apoptotic cell death ${ }^{(22)}$.

Few studies have been done to illustrate the cytotoxic effect of antidepressant. So this study aimed to examine the possible impact of long term administration of antidepressant duloxetine on the oral mucosa and salivary glands in tongue of albino rats using routine hematoxylin\&eosin and Masson' trichrome stains for histopathological evaluation. Immuno-histochemical investigations of Bax monoclonal antibodies for detection of any apoptotic changes in the above mentioned tissues. 


\section{MATERIALS AND METHODS}

Drug preparation: The antidepressant drug duloxetine (Cymbalta),was purchased from Eli Lilly medical Company which supplied in capsules form with concentration 30mg/tab. These capsules were dissolved at distilled water to a concentration of $10 \mathrm{mg} / \mathrm{ml}$.

Animals and experimental Design: Twenty adult male albino rats, weighting 200-225 gram body weight were used in this investigation. They were housed in rat cages (five rats per cage), and labeled with numerical numbers and kept in well ventilated animal house at the faculty of dentistry, Suez Canal University, at temperature $27-30^{\circ} \mathrm{C}$, 12 hours natural light and 12 hours darkness. The rats were supplied with standard natural diet and tap water adlibitum throughout the whole experimental period which lasted for three months. The animals were divided into two equal groups, ten animals each as followed: Group 1: served as control group which received distilled water in comparable volume to treated group with the same route of administration. Group 2: served as treated group which received duloxetine dissolved in distilled water in a daily oral dose of $10 \mathrm{mg} / \mathrm{kg}$ body weight (b.w.) using a curved metallic oro-pharyngeal tube ${ }^{(23)}$. At the end of the experiment, the rats were euthanized by cervical dislocation.

Tissue processing and staining: The tongues were dissected out, fixed in $10 \%$ freshly prepared neutral buffered formalin, and processed for being embedded in paraffin. 4-5-micron-thick sections were dissected. Then, they were stained with hematoxylin and eosin to detect any possible histological changes, Masson' trichrome stain for collagen detection. Then photographed by E-330 Olympus digital camera.

For immuno-histochemical (IHC) evaluation (for detection of any apoptotic changes in the tongue tissues), $5 \mu \mathrm{m}$ sections were cut and mounted on positively-charged slides. The immunostaining were performed using rabbit polycolonal mouse antibody to Bax, purchased from Santa Cruz Biotechnology, Cat.No. sc-526. The steps of IHC were followed according to manufacturer's instructions.

\section{Immuno-histochemistry Quantification:} Digital image analysis was done, using software of image analyzer computer system (image J / Fiji 1.46). The area of the screen was measured by digitizing the slides under 200X objective magnification. The pixel intensity of the colour, Bax labeling index (The number of Bax positive cells divided by the total cells) was estimated and treated statistically.

Statistical analysis: for entering the data, Microsoft excel 2013 was employed, and for analyzing the data, the SPSS (version 24) was utilized.The median \pm standard deviation was utilized as simple descriptive statistics for summarizing the skewed quantitative data, and for summarizing the qualitative data, frequencies were employed. Further, for comparing the quantitative data which is distributed non-normally, Mann-Whitney test was utilized. When $\mathrm{P}<0.04$, differences were perceived as statistically significant.

\section{RESULTS}

\section{I- Histopathological results}

\section{a) Hematoxylin\& eosin and Masson' trichrome stains:}

\section{Control group}

The mucosal membrane of the dorsal and ventral surfaces of the body of the tongue is formed of stratified squamous epithelium and underlying lamina propria formed of dense collagen arranged in bundles with connective tissues cells of which the fibroblasts are the predominant type. The collagen fibers revealed normal distribution with strongly positive staining reactivity to Masson's trichrome stain (Figure3; A). The lamina propria is moderately vascular and well innervated. 
The dorsal surface showed different types of papillae including the filliform, fungiform papillae with a taste bud presented on its top surface and circumvallate papillae with a lot of taste buds are found in the side walls of the papillae and furrows. Lingual salivary are also seen among the lingual muscles fibers showed normal histological architectures (Fig.1).

\section{2-Group II}

The histological examination of the tongue obtained from animals subjected to daily oral dose $10 \mathrm{mg} / \mathrm{kg} \mathrm{b}$. w. of duloxetine for 3 months presented noticeable atrophic and degenerative changes that involved the surface epithelium and lamina propria of both the dorsal and ventral surfaces as well as the lingual glands.
The surface epithelium presented abnormal cytoplasmic vacuolization of some epithelial cells, single cells showed typical signs of apoptosis manifested as their shrinkage and detachment from the neighboring cells, appeared as a round or oval mass with dark eosinophilic cytoplasm with pyknotic, fragmented or absent nucleus (Fig 2; C,D).

The filliform papillae showed deformation, decrease in length and number, their epithelial covering was atrophic, areas devoid the epithelial ridges were found. Most of them showed flattening, loss of their characteristic conical shape, with evident hyperkeratosis (Fig. 2; A,C).

The fungiform and circumvallate papillae showed signs of atrophy in their epithelial covering, their lamina propria and taste buds. The later were shrunken, degenerated or completely lost (Fig. 2; A, B).

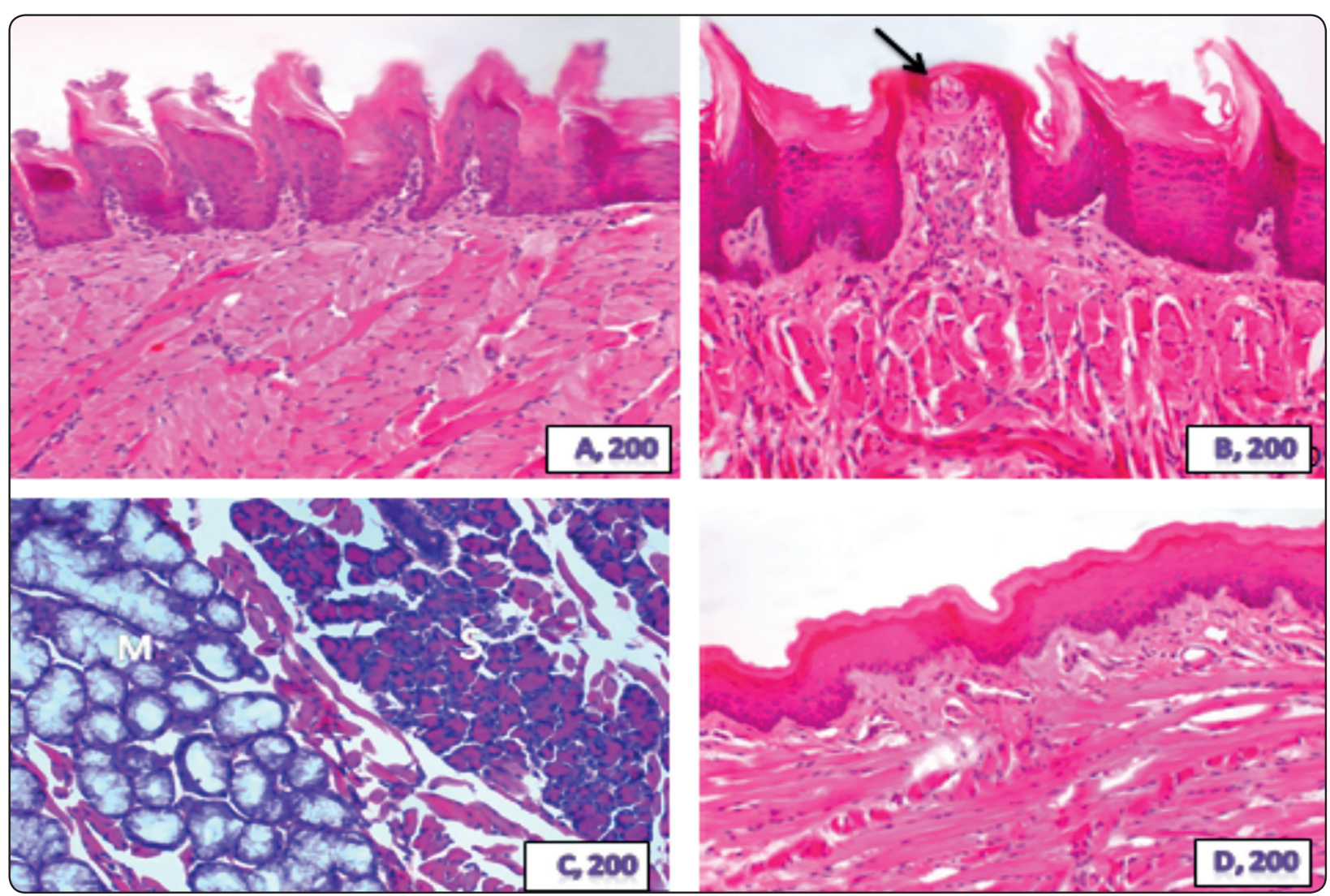

Fig. (1): A photomicrograph of the tongue of control group rats showing of the dorsal surface (A) filliform papillae. (B) fungiform papilla with taste bud (arrow). (C) Serous (S) and mucous (M) acini of the lingual salivary glands. (D) the mucous membrane of the ventral surface (HX\&E. orig. mag.200). 
The underlying lamina propria showed degeneration and dissociation of collagen that revealed weakly positive staining reactivity to Masson's trichrome stain (Fig. 3; B) with mild inflammatory cell infiltration. The blood vessels showed dilatation with blood stagnation.

The lingual glands also suffered from marked degenerative changes in the acini and ducts of both serous and mucous glands. The serous acinar cells presented with intracytoplamic vacuolization. The mucous acinar cells were also seriously affected with cystic transformations. The ducts of both serous and mucous glands were atrophic with marked flattening of their epithelial lining and collection of stagnant secretions forming cyst- like structures (Fig 2.F,G).

The ventral surface of the tongue showed marked atrophic and degenerative changes represented by thin epithelium, atrophic with intracellular cytoplasmic vacuolizations and some cells showed apoptosis. The lamina propria showed dissociation and degeneration of the collagen, presented weakly positive staining reactivity with Masson's trichrome stain (Fig 3; C). The blood vessels showed massive dilatation with blood stagnation( Fig2; H,I).

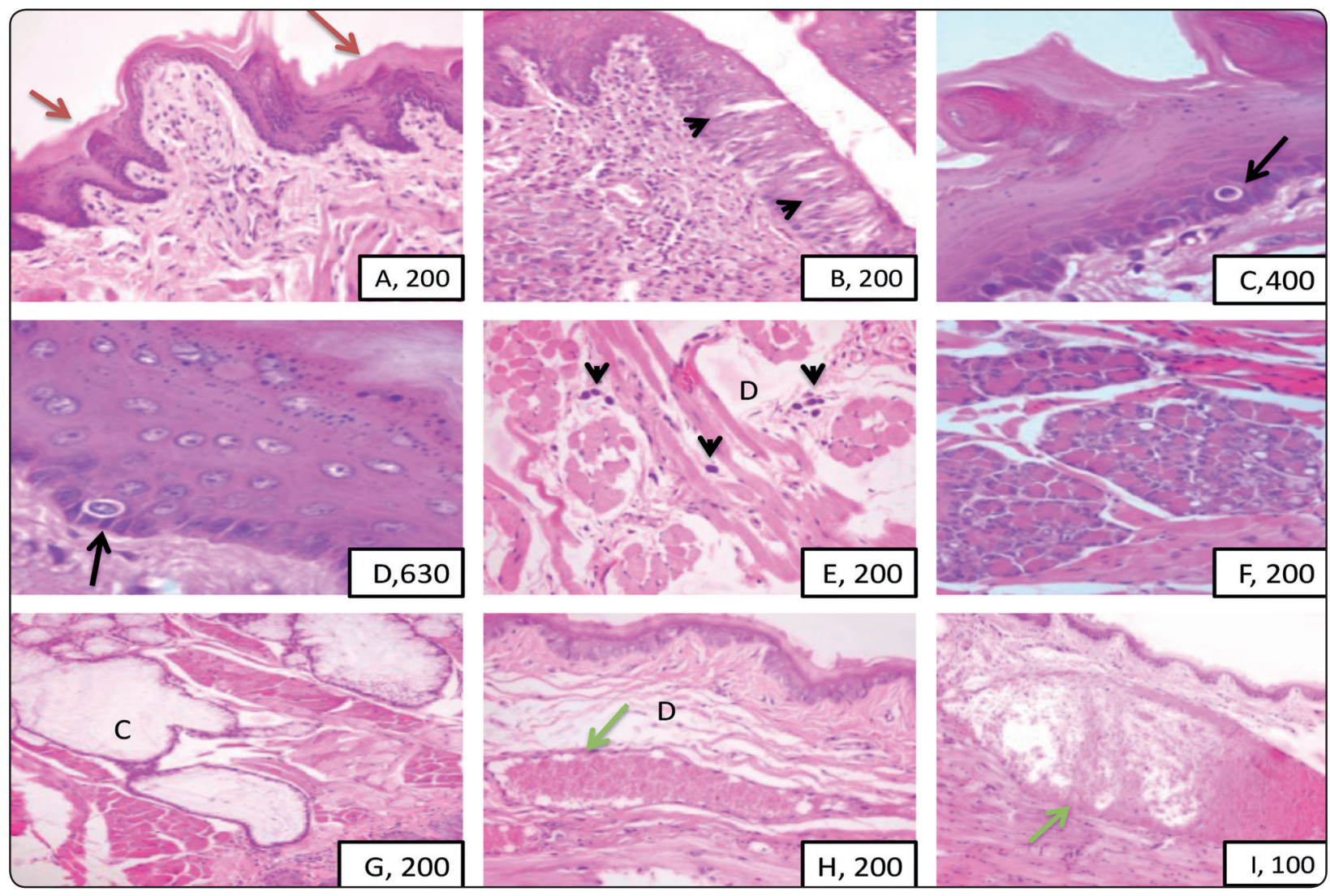

Fig. (2): A photomicrograph of the dorsal and ventral surfaces of tongue of rats received duloxetine for 3 months showing (A) deformed and degenerated filliform (red arrows) and fungiform papillae. (B) atrophied circumvellate papillae with degenerated taste buds (arrow heads). (C, D) epithelial cells showing intracellular cytoplasmic vacuolizations and some cells showed apoptosis (arrows). (E) lamina propria showing degeneration (D) and phagocytic cells (arrow heads). (F) serous acinar cells with cytoplasmic vaculization. $(\mathrm{G})$ cystic transformation of mucous acini $(\mathrm{C})$. (H,I) extreme atrophy of the surface epithelium of the ventral surface with degeneration (D) of the lamina propria and massive dilatation of the blood vessel that engorged with RBCs (green arrows). (HX\&E. orig. mag 200,400, 630,200,100) 


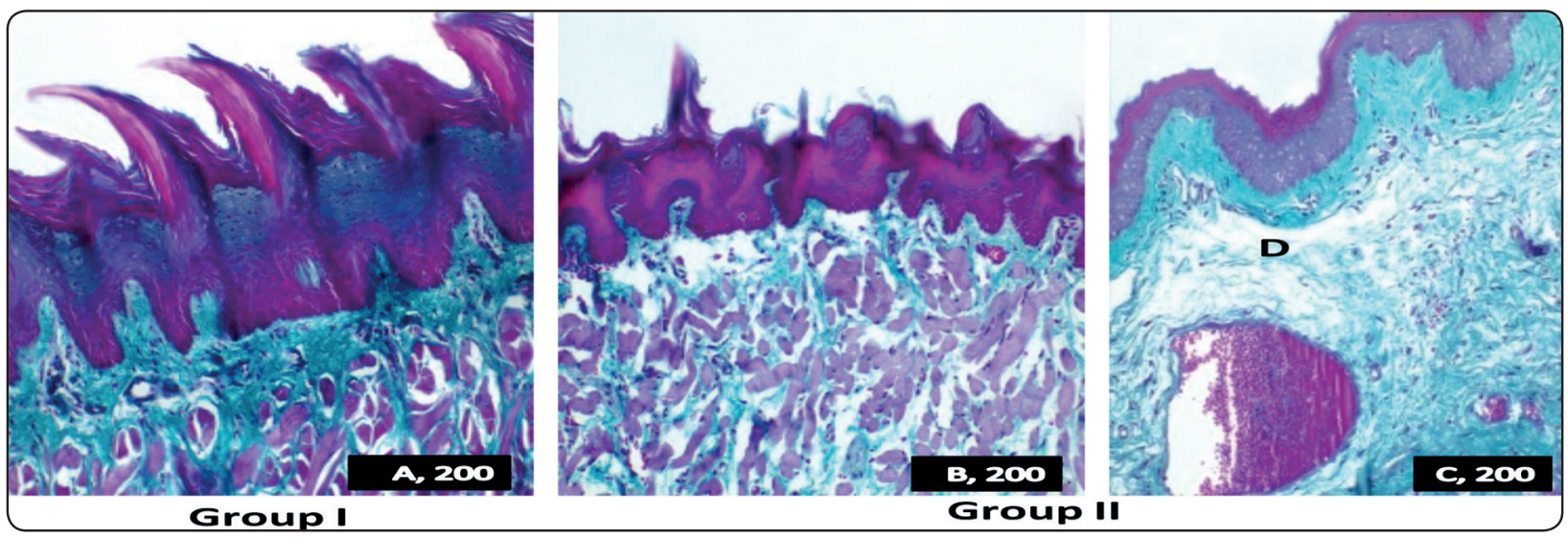

Fig. (3): A photomicrograph of the tongue tissue of different groups stained with the Masson's trichrome stain. A- control group showing strongly positive reactivity of the collagen fibers with Masson's trichrome stain. B,C- animals of group II treated with duloxetine for 3 months showing degeneration and dissociation of collagen revealed weaker positive staining reactivity than Masson's trichrome stain (Masson's trichrome , origin mag. 200)

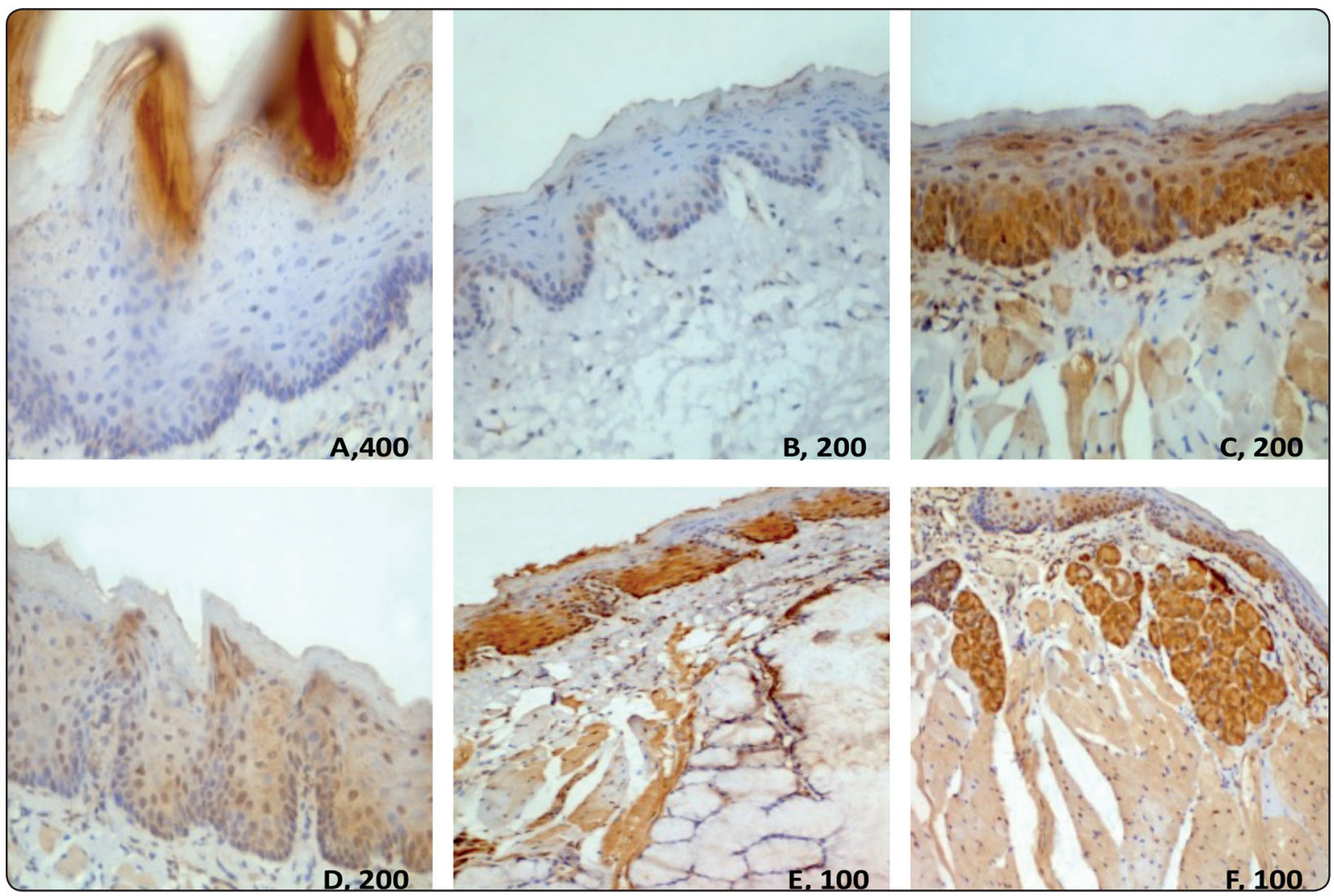

Fig. (4): A photomicrograph of the tongue of both control and experimental rats incubated with Bax monoclonal antibody, A, B: showing light expression of Bax limited to superficial layers and keratinized layer in the control group, the fibroblasts in the underlying lamina propria and the blood vessels. C,D,E,F: showing heavy cytoplasmic expression of Bax in the duloxetine - treated group in all layers of keratinocytes, fibroblasts and blood vessels of the lamina propria and lingual salivary gland. (Orig. Mag.400, 200,100). 


\section{II- Immuno-histochemical results:Immuo-histo- chemical localization of (Bax):}

Examination of Bax expression (brown cytoplasmic staining) in normal control tissue showing weak expression localized to the superficial cell layers of epithelium, the fibroblasts of the underlying lamina propria and blood vessels (Fig.4; A,B). While samples treated with duloxetine revealed heavy expression of Bax in all layers of keratinocytes, fibroblasts and blood vessels of the underlying lamina propria as well as acinar and ductal cells of lingual salivary glands (Fig.4; C,D,E,F).

III- Statistical results: Mann-Whitney test showed a high statistical significant difference between both groups regarding the mean (P-value $=0.002)$ (table 1), among Bax expression for the duloxetine and control groups (figure 5).

TABLE(1): The median score and $\mathrm{P}$ value of Bax expression in control and treated group

\begin{tabular}{|c|c|c|c|}
\hline Group & $\mathrm{N}$ & Median $\pm(\mathrm{SD})$ & $\begin{array}{c}\mathrm{P} \\
\text { value }\end{array}$ \\
\hline Bax (control) & 10 & $21.61300 \pm 9.139242$ & \\
\hline Bax (treated group) & 10 & $190.64800 \pm 46.356804$ & \\
\hline
\end{tabular}

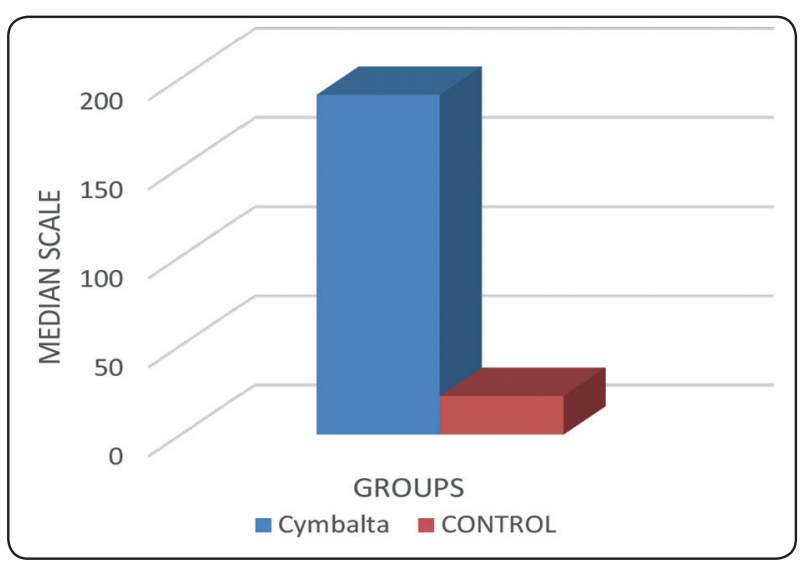

Fig. (5): Showing the median score of Bax expression in control (red bar) and duloxetine (Cymbalta) -treated group (blue bar).

\section{DISCUSSION}

Described as being SNRI (a selective serotonin and norepinephrine reuptake inhibitor) antidepressants, the duloxetine is utilized for the treatment of pain, depression, and anxiety. Therefore, it is one of the most commonly used medications in adults.

The histological results of the present study revealed that long term administration of duloxetine resulted in noticeable atrophic and degenerative changes in the surface epithelium and lamina propria of both the dorsal and ventral surfaces of the tongue as well as the lingual salivary glands in the adult albino rat. Light microscopic examination of the surface epithelium presented abnormal cytoplasmic vacuolization of some epithelial cells, single cells showed typical signs of apoptosis appeared as a round or oval, shrinked, the cytoplasm was characterized by density and the organelles were considered as being packed more tightly. The nucleous showed pyknosis as the result of chromatin condensation, or karyorrhexis. Our finding go with Hacker ${ }^{(24)}$ who described the morphology of apoptosis. In the current study, mononuclear cellular infiltration was detected. This current observation concur with Hart, et $\mathrm{al}^{(25)}$ who studied phagocytosis of apoptotic cells.

Mitochondria are the cellular powerhouse of eukaryotic cells, and they also regulate cell function through oxidative stress and apoptosis. The current results were consistent with previous studies done by Youssef ${ }^{(26)}$, Souza et $\mathrm{a}^{(27)}$ and Elmorsy et $\mathrm{al}^{(28)}$ who demonstrated that fluoxetine harmfully affected the histological structure of the pancreatic and hepatic cells. They come backed this toxic effect to the mitochondria and stated that mitochondrial damage led to ATP reduction with successive failure of biosynthesis, maintenance of the cytoskeleton, signaling and ion transport, membrane pumps, oxidative stress and induction of apoptosis. Consequently, in our opinion the marked degenerative changes, cystic transformations and collection of stagnant secretions in the acini and ducts of the lingual glands are due to ATP reduction and de- 
pletion of cellular energy used for transport of secretions.

Moreover, mitochondria also generate reactive oxygen species (ROS) that may have a toxic effects on cells. Vitally, increase the production of ROS lead to damage to mitochondrial, cytoplasmic proteins, lipids and nucleic acids. Our result go with Swager and Morgan ${ }^{(29)}$ who stated that among all side effects that are reported of antidepressants and antipsychotics, dry mouth is frequently the most prevailing complaint. Khan et $\mathrm{al}^{(30)}$ reported that duloxetine antagonizes the muscarinic receptor and cause anticholinergic adverse effect.

Previous study done by Navazesh and et $\mathrm{al}^{(31)}$ showed that through the alteration of neural pathways, which induce the secretion of salivary gland through the prohibition of the cholinergic signals within the salivary tissues, resulting in diminishing fluid excretion by glands, xerostomia is induced. According to another study, there is an anticholinergic and antimuscarinic action of SNRI antidepressants that leads to blocking parasympathetic system action and inhibits acetylcholine influence on the receptor of salivary glands thus induce dry mouth by prevalence of the sympathetic nervous system on the blocked parasympathetic system ${ }^{(32)}$. Accordingly we suggested that the degenerative changes that occurred in the lingual mucosa after long administration of duloxetine as result of decreasing in the salivary flow that leading to decrease in lubrication of the oral mucosal tissues and enhance their atrophy and inflammation.

Massive dilation of blood vessels of the lamina propria of the experimental group was a constant feature in our results. This arteriovenous dilation may lead to engorgement and stagnation of blood and decrease in its flow, resulting in hypoxia and ischemia of the tongue tissues. In our opinion, this hypoxia and ischemia may have played a role as a cause of the present structure deterioration of the epithelium, lamina propria and salivary glands. According to several studies, cell injury, as well as cell death is mediated by hypoxia through apoptosis, oxidative stress, acidosis, and inflammation ${ }^{(33)}$. On the other hand, several other studies showed that apoptosis is induced by hypoxia through the inducement of calcium overload, increased oxygen free radicals, mitochondrial damage, and the increase in HIF (hypoxia-inducible factor) expression ${ }^{(34,35)}$. As a result, hypoxia is closely related to apoptosis and this is concur with our investigation.

Microvesicles (MVs) are perceived as cell-cell communication's new regulators and that's why much attention has been paid to them recently, and they are considered as membranous vesicles with a $0.1-1-\mu \mathrm{m}$ diameter. Several types of cells can release them when they are injured, activated or undergone apoptosis. These MVs can carry bioactive molecules shed from injured mother cells and transfer it into adjacent normal cells, thereby causing a series of cellular changes including cell apoptosis. It can be seen that cell injury is not entirely the result of direct stimulation of cells by hypoxia or other stimuli. It is also possible that injured cells indirectly act on peripheral normal cells by releasing the MVs, which contain substances that promote injury and apoptosis. It is the MVs that mediate the amplification of damage ${ }^{(36,37)}$.

In the current study immuno-histochemical results revealed that Bax expression of the control group was restricted to superficial layers of the epithelium.This result was in agreement with Assis, et $\mathrm{al}^{(38)}$. While alteration in the Bax expression were detected, Bax get over expressed in the lingual mucosa of duloxetine treated group all over the epithelial layer and lamina propria, these results get confirmed statistically as there was high significantly difference ( $\mathrm{P}$-value $=0.002$ ) between the two groups.

Under the circumstances of stress, a conformation change is undergone by Bax where the mitochondrion membrane is translocated, resulting in releasing cytochrome $\mathrm{c}$, which in turn promoting CASP3 activation, leads to triggering apoptosis ${ }^{(39)}$. 
This is consistent with our findings.

Finally, it could be postulated that these apoptotic, cytotoxic and degenerative changes indicated disturbance in cellular homeostasis and cellular failure as result of long term administration of duloxetine.

\section{CONCLUSION}

Although duloxetine being useful drug in treatment depression, anxiety and severe pain, it harmfully affected the histological structure of oral mucosa and salivary glands of the tongue in adult rats. Accordingly using of these drugs should be under supervision and strong caution

\section{REFERENCES}

1. American Psychiatry Association (APA): Diagnostic and Statistical Manual of Mental Disorders (DSMIV-R). 4ed. Washington DC: American Psychiatry Association; 1994.

2. Gregor, H.: Pathophysiology of depression: Do we have any solid evidences of interest to clinicians?World Psychiatry. 2010 ; 9: 155-161.

3. Belmaker, R.H. and Agam, G.: Major depressive disorder. N Engl J Med. 2008; 358: 55-68.

4. Fountolakis, K.N.; O'Hara, R. and Apostolosacovides , C.: Unipolar late onset depression, A comprehensive review . Ann.Gen. Hos. Psych. 2003 2:11- 14

5. Clayton, A. H.; Pradko, A. F.; Croft, H. A., Montano, C. B.; Leadbetter, R. A.; Bolden-Watson, C.; Bass, K. I.; Donahue, R. M.; Jamerson, B. D. and Metz, A.: Prevalence of sexual dysfunction among newer antidepressants. J Clin Psychiatry. 2002; 63:357-366.

6. Masand, P. S. and Gupta, S.: Long-term side effects of newer-generation antidepressants: SSRIs, venlafaxine, nefazodone, bupropion, and mirtazapine. Ann Clin Psychiatry. $2002 ; 14: 175-182$

7. Richelson, E.: Interaction of antidepressants with neurotransmitter transporters and receptors and their clinical relevance. J Clin Psychiatry. 2003; 64: 5-13.

8. Cashman, J.R. and Ghirmai, S.: Inhibition of serotonin and norepinephrine reuptake and inhibition of phosphodiesterase by multi-target inhibitors as potential agents for depression. Bioorganic \& Medicinal Chemistry. 2009; 17: 6890-7.
9. Iyengar, S.; Webster, A. A.; Hemrick-Luecke, S. K.; Xu, J. Y and Simmons, R. M: Efficacy of duloxetine, a potent and balanced serotonin-norepinephrine reuptake inhibitor in persistent pain models in rats. J. Pharmacol. Exp. Ther. 2004; 311: 576-84.

10. Russell, I. J.; Mease, P.J.; Smith, T. R; Kajdasz, D. K.; Wohlreich, M. M; Detke, M. J. and Walker, D. J.: Efficacy and safety of duloxetine for treatment of fibromyalgia in patients with or without major depressive disorder: results from a 6- month, randomized, double-blind, placebo-controlled, fixed-dose trial. Pain. 2008; 136: 432-444.

11. Goldstein, D. J.; Lu, Y.; Detke, M. J.; Lee, T. C. and Iyengar, S.: Duloxetine vs. placebo in patients with painful diabetic neuropathy. Pain. 2005;116:109-118.

12. Wernicke, J. F.; Pritchett, Y. L.; D’Souza, D. N.; Waninger, A.; Tran, P.; Iyengar, S. and Raskin, J.: A randomized controlled trial of duloxetine in diabetic peripheral neuropathic pain. Neurology. 2006; 67: 1411-1420.

13. Skljarevski, V.; Zhang, S.; Desaiah, D.; Alaka, K. J; Palacios, S. Miazgowski, T. and Patrick K.: Duloxetine versus placebo in patients with chronic low back pain: a 12-week, fixed-dose, randomized, double-blind trial. J Pain. 2010; 11:1282-90.

14. Michele. D. M.; Daniela.A.; Vittorio, S.; Marco. G. R. and Giulio F.: Burning Mouth Syndrome Responsive to Duloxetine: A Case Report, Pain Medicine. 2011; 12: 466-469.

15. Yeon-Dong, K.; Ji-Hye L. and Jee-Hoon S.: Duloxetine in the treatment of burning mouth syndrome refractory to conventional treatment: A case report. Journal of International Medical Research 2014; 42: 879-883.

16. Nagashima, W.; Kimura, H.; Ito, M.; Tokura, T.; Arao, M.; Aleksic B.;Yoshida, K.; Kurita, K. and Ozaki, N.: Effectiveness of duloxetine for the treatment of chronic nonorganic orofacial pain. Clin Neuropharmacol 2012; 35: $273-277$.

17. Nerella, N. K.; Mamatha, G. P. and Rajeshwari, G. A.: Modified schirmer test-A screening tool for xerostomia among subjects on antidepressants. archives of oral biology. 2014 ; 59: 829- 834 .

18. Eischen, C. M.; Roussel, M. F.; Korsmeyer, S. J. and Cleveland, J. L.: Bax loss impairs Myc-induced apoptosis and circumvents the selection of p53 mutations during Myc-mediated lymphomagenesis. Mol. Cell. Biol. 2001;21: 7653-7662. 
19. Norbury, C.J. and Hickson, I. D.: Cellular responses to DNA damage. Annu Rev Pharmacol Toxicol. 2001; 41:367-401.

20. Edlich, F.; Banerjee, S.; Suzuki, M.; Cleland, M. M.; Arnoult, D.; Wang ,C.; Neutzner, A.; Tjandra, N. and Youle, R. J.: Bcl-x (L) retrotranslocates Bax from the mitochondria into the cytosol. Cell. 2011; 145:104-116.

21. Krajewski, S.; Krajewska, M.; Shabaik, A.; Miyashita, T; Wang, H. G. and Reed, J. C: Immunohistochemical determination of in vivo distribution of Bax, a dominant inhibitor of Bcl-2. Am J Pathol. 1994; 145:1323-1336.

22. Korsmeyer, S J,; Shutter, J. R.; Veis, D. J.; Merry, D. E. and Oltvai, Z. N.: Bcl-2/Bax: a rheostat that regulates an anti-oxidant pathway and cell death. Sem Cancer Biol. 1993; 4:327-332

23. Patel, S.; Kale, P.; Addepalli, V.; Sarker, A. and Savai, J.: Effect of a combination of duloxetine with hydroxyzine on experimental models of anxiety in mice. Indian J. Pharmacol. 47: 173-176. 2015.

24. Häcker, G.: The morphology of apoptosis. Cell Tissue Res. 2000; 301:5-17.

25. Hart, S. P.; Dransfield, I. and Rossi, A. G.: Phagocytosis of apoptotic cells. Methods. 2008;44:280-5.

26. Youssef , S.: Effect of Fluoxetine on the Pancreas of Adult Male Albino Rats and the Possible Protective Role of Omega-3: Light and Electron Microscopic Study. International Journal of Clinical and Developmental Anatomy. 2017; 3: 45-56

27. Souza, M.,E.; Polizello, A. C.; Uyemura, S.,A.; Castro, S.O. and Curti C. : Effect of fluoxetine on Rat liver mitochondria. Biochemical Pharmacology 1994;48:535-541

28. Elmorsy, E; Al-Ghafari, A.; Helaly, A.N.M.; Hisab, A. S.; Oehrle, B. and Smith, P. A.: Editor's highlight therapeutic concentrations of antidepressants inhibit pancreatic betacell function via mitochondrial complex inhibition. Toxicol. Sci. 2017; 158: 286-301.

29. Swager, L.W and Morgan, S.K.: Psychotropic-induced dry mouth: don't overlook this potentially serious side effect. Current Psychiatr. 2011;10:54-8
30. Khan, A.; Bose, A; Alwxopoulous, G. S. ; Gommoll, C.; Li, D. and Gandhi, C.: Double blind comparison of escitalopram and duloxetine in acute treatment of major depressive disorder. Clin Drug Investig. 2007; 27: 481-492.

31. Navazesh, M.; Brightman, V. J and Pogoda, J. M.: Relationship of medical status, medications, and salivary flow rates in adults of different ages. Oral Surg Oral Med Oral Pathol Oral Radiol Endod 1996;81:172-6

32. McCorry, L.; : Physiology of the autonomic nervous system. Am J Pharm Educ. 2007;71:78.

33. Kubasiak, L.A; Hernandez, O. M.; Bishopric, N. H. and Webster, K. A: Hypoxia and acidosis activate cardiac myocyte death through the Bcl-2 family protein BNIP3. PNAS October 1, 200299 (20) 12825-12830.

34. Webster, K.A.: Mitochondrial membrane permeabilization and cell death during myocardial infarction: roles of calcium and reactive oxygen species. Future Cardiology. 2012; 8: 863-884.

35. Chen, R.; Lai, U. H.; Zhu, L.; Singh, A.; Ahmed, M. and Forsyth, R.: Reactive Oxygen Species Formation in the Brain at Different Oxygen Levels: The Role of Hypoxia Inducible Factors. Front Cell Dev Biol. 2018; 6: 132.

36. Ying Guo,Y.; Tan,J.; Sun, Z. and Zhang,Q.: Effects of Microvesicles on Cell Apoptosis under Hypoxia Oxidative Medicine and Cellular Longevity.2019, Article ID 5972152, 11 pages.

37. Lee, T.H.; D’Asti, E; Magnus. N; Al-Nedawi K.; Meehan, B. and Rak, J.: Microvesicles as mediators of intercellular communication in cancer-the emerging science of cellular 'debris', Seminars in Immunopathology. 2011; 33: 455-467.

38. Assis, G.; Ceolin, D.; Marques, M.; Salvadori, D. and Ribeiro.: Cigarette smoke affects apoptosis in rat tongue mucosa: role of bcl-2 gene family.J Mol Hist.2005; 36:483-489.

39. Czabotar, P. E.; Lee, E. F.; Thompson, G. V.; Wardak, A Z.; Fairlie W. D. and Colman, P. M.: Mutation to Bax beyond the $\mathrm{BH} 3$ domain disrupts interactions with prosurvival proteins and promotes apoptosis. J. Biol. Chem. 2011; 286:7123-7131. 